DOI: 10.20472/TEC.2019.008.001

CHIZUKO AIBA

Tokyo Denki University, Japan

JUNJI IZUMI

Shibaura Institute of Technology, Japan

\title{
DO PRODUCTIVE SKILLS OF LANGUAGE ENHANCE LEARNERS' COGNITIVE ABILITY? : AN EXPERIMENTAL STUDY OF SOFT CLIL TO TECHNOLOGY MAJORS
}

\begin{abstract}
:
STEM (Science, Technology, Engineering, and Mathematics) is one of the subject areas which are being taught in Japan by employing CLIL (Content and Language Integrated Learning). Within the framework of teaching methodology, output tasks are considered to be effective in helping learners' cognition with the target language. UBM (Usage-based model) supports this idea as learners can use the language more in the output tasks to explain and illustrate what they are paying attention to. In this study, the hypothesis is examined by teaching English in CLIL methodology to technology majors at a Japanese university. Throughout the semester, the output-focused group $(N=54)$ is oriented to output tasks, while the input-focused group $(\mathrm{N}=24)$ is focused on reading material. Both groups are taught by the same English teacher in Soft CLIL. Their improvement of understanding of English involved with logical thinking can be measured by pre- and post-GJTs (Grammaticality Judgement Tests) which contain causal relationship with conjunctions. A t-test of the results shows that both groups shows significant differences between pre- and post-tests (input: $t=1.7633$, $p=0.04181$; output: $t=1.9017, p=0.03491$ ). However, no significant differences between the input group and output group are observed in both pre- and post-tests.
\end{abstract}

\section{Keywords:}

STEM, CLIL, UBM, GJT

JEL Classification: 120, 123 

Introduction

In higher education, STEM (Science, Technology, Engineering, and Math) is becoming a more and more important field as the outcome of teaching and learning are evaluated quantitatively. These subjects are put more emphasis than before since they are directly applied to industries.

In non-English speaking countries, various types of English communication skills are incorporated into the curriculum in higher education as requirements since the economic globalization has widely penetrated into students' working lives after their graduating from universities. As such, their standardized test scores are one of the critical factors that could determine their career opportunities.

As a result, STEM and English education are becoming closer to each other in these countries. On the one hand, content professors who have subject knowledge of STEM teach these subjects in using the English language in their subject course, known as EMI (English as Medium instruction). On the other, teachers of English language teach English by incorporating STEM content in their language classes. Indeed, relevant classes fit in any point of the continuum with these two extremes (Lin, 2019).

One author of this paper (Aiba) has a long experience in teaching English to technology majors at a university in Japan while conducting action research of the CLIL (Content Language Integrated Learning) approach (Coyle et al., 2010) in a primary school context. This means that, as a non-native English teacher, she has been implementing a Soft CLIL (Bentley, 2010) by incorporating subject materials, which are sometimes related to science and math.) The primary purpose of Soft CLIL is language-focused learning, while that of Hard CLIL is content focused learning.

The other author (Izumi) has been attempting to apply Soft CLIL to his classroom at a technology university for several years. At first he tried bits of EAP (English for Academic Purpose) such as note-taking and summary writing (Deller \& Price, 2007), then incorporated Soft-CLIL in some of his classes by CLIL since this approach fits his students who can tackle cognitive challenges of their interests but need much language help in the EFL (English as Foreign Language) environment. Such teachers' scaffolding is intended to support input and output so that learners can stand alone (Dale et al., 2010).

CLIL has a solid framework which comprises 4Cs: Content, Communication, Cognition, Culture. The primary focus of this paper is the relationship between the two key components of Communication and Cognition.

\section{$2 \quad$ Literature Review}

Bloom's taxonomy underpins Cognition of CLIL, where the lower order thinking (memorizing, understanding, applying) are supposed to develop into higher-order thinking (analyzing, evaluating, creating) (Krathwohl, 2002). Hypothesizing, for example, along with predicting and inferring, is at the second tier from the top of higher-order thinking skills, where students combine ideas to form a new one.

CLIL research communities have attributed the interdependence of Communication and Cognition to academic language functions often used in CLIL classrooms, which mostly corresponds to the thinking skills in Bloom's Taxonomy. Based on her introspection, a researcher shows connections between the cognition and communication, by listing the lexical verbs and phrases. For example, hypothesizing function goes with the words, assume, guess, hypothesize, imagine, ....., and with the phrases let's think /say/assume/imagine ... (Dalton-Puffer 2007, pp.160-167). For her, the medium which links Cognition with Communication is the use of specific words or phrases.

Empirical data drawn from CLIL practices seem to support the strong relationship between the use of academic functional languages and the development of thinking skills. In a Japanese secondary 
school, the science CLIL group achieved much higher than non-CLIL group in learning first and third conditional. The primary factor for the group's success was abundant use of target grammar in peer discussion with occasional scaffolding, rather than teacher-led explicit grammar instruction (Kashiwagi et al., 2019).

The theoretical background of this specific practical research heavily relies upon the usage-based model (UBM). In contrast to Chomskian generative approach, UBM regards language learning as the process that the units of language emerge from the communicative process (Bybee \& Beckner, 2010). In short, language is not an innate structure but is leaned by actual language use in actual communicative events (Tomasello, 2000).

Although UBM has been developing in L1 researches, it can be useful to understand L2 learning. In the above CLIL practice, students' discussion before the experiments is highlighted because students are given opportunities to confirm or disconfirm their hypotheses in groups, where units of language emerge not sufficiently but involving the target grammar. This is compared to a joint-attentional scene in which children acquire L1 through social-cognitive interaction, sometimes with gesturing (Tomasello, 1999, pp.94-133).

The application of UBM to L2 learning helps us to understand the connection between CLIL academic functional language and student's cognitive development. However, it seems to be premature to conclude that only language use stimulates thinking. First, UBM accounts for verb constructions with word order and case construction (Tomasello, 1999, pp.134-160), but does not contend any on more complicated construction with two clauses. Second, CLIL researches under the influence of UBM do not make it clear what sorts of use are effective to the cognitive development of learners.

\section{Research Question}

Following the above critical review of the literature, the current study addresses the following research questions:

RQ1. Do students improve cognitive ability through Soft-CLIL practices?

Rather than hypothesizing in higher-order thinking, we will look at the more fundamental thinking, i.e, a logical connection. In Bloom's taxonomy, it is categorized into both lower-order thinking and higher-order thinking. More specifically, the precise understanding of the cause-result relationship is focused.

RQ2. Which of input or output is more effective on the learners understanding of causal relationship?

Input and output usually co-exist in language classrooms. However, if we look at the teaching practice of a course or of a lesson only, it often leans on either side of input or output, so the input-focused class and the output-focused class will be addressed.

\section{$4 \quad$ Method}

At a technology university in Japan, 78 sophomore students participated in this study. They are divided into two groups named the input-focused group and output-focused group (input and output hereafter). The input group has 54 students who enrolled in two classes of the course called Reading IA, and the output group has 24 students enrolled in two classes of the course called Technical English IA. These four classes are taught by one of the authors (Izumi) on Wednesdays every week. The participants wrote their consent to allow their responses to be analyzed quantitatively in the current study.

The two courses are comparable because both are assigned vocabulary quizzes of target lists uploaded on Quizlet, extensive pair- and group works, and more importantly, the soft-CLIL methodology. Typical lessons of these courses are as follows. 
The input group was given an outlining task of scientific articles contained in the reading textbook (Cleary, K., Nozaki, Y., \& Matsumoto, K., 2012), pair speaking task of retelling the article based on the outline, following comprehension check $Q \& A$, and discussion on relevant topics. To help these activities, handouts such as lists of note-taking signs and phrases for discussion are distributed. Also, students are assigned to read one graded reader by the end of the course.

The output group learns the vocabulary, reading, and listening sections of a unit of the textbook (Phillips, T., Hitomi, K., \& Yubune, E.,2005) as language scaffolding in the first half of a lesson. Then an original output task to use the language they learned from the textbook is implemented in the second half. In this task, students take note, talk in pair or group, or occasionally solo- or group class presentations. Language scaffolds are given as a sample video and phrases for presentation for this task.

Explicit grammar teaching was not foregrounded in both courses because they are task- oriented as described above. As a result, mid-term and final tests do not contain an exclusive grammar section. However, both textbooks contain reasonable grammar explanations, including conjunctions to express a causal relationship to be measured in the current study.

The two-group pre- \& post-test design was employed to measure effects on input and output groups between the beginning of the term and the end of the term. The measurement tool was the same timed grammaticality judgment tests (GJTs) (Ellis, 1991; Godfroid, Loewen, Jung, Park, Gass, \& Ellis, 2015) for both groups. Each test contains eight sentences with two clauses connected by conjunction so or because, two of which are extracted from the textbook for the input group, two from the textbook for the output group, and the four are from the graded reader corpus (Compleat Lexical Tutor). Students have to answer if these sentences are correct or incorrect by judging the logical connection between the two clauses of each sentence.

\section{$5 \quad$ Results and Discussion}

The descriptive statistics of the timed GJT results and graphic comparison of the two groups are shown in Table 1 and Figure 1. Both the input group and output group show moderate increases of means. A paired one-sided t-test with $\mathrm{R}$ shows significant differences between pre-test and post-test for both input group and output group (Input: $t=1.7633, d f=53, p=0.04181<0.05$; Output $t=1.9017, d f=23, p=0.03491<0.05)$.

However, no significant differences between groups are observed in both pre-test and post-test by performing a Welch two sample t-test (two-sided) with $\mathrm{R}$. (Pre-test: $t=0.66482, d f=57.271$, $\mathrm{p}=0.5088>>0.05$; Post-test: $t=0.39754, d f=62.899, p=0.6923>>0.05)$

Table 1

Descriptive Statistics of Timed GJT Results

\begin{tabular}{lcrrrrrrrr}
\hline & \multicolumn{3}{c}{ Pre-test } & \multicolumn{4}{c}{ Post-test } \\
\cline { 2 - 9 } & \multicolumn{1}{c}{$\mathrm{M}$} & $\mathrm{SD}$ & Min & Max & $\mathrm{M}$ & $\mathrm{SD}$ & Min & Max \\
\cline { 2 - 9 } Input group $(\mathrm{N}=54)$ & 5.148148 & 1.68688 & 1 & 8 & 5.666667 & 1.613621 & 2 & 8 \\
Output group $(\mathrm{N}=24)$ & 4.916667 & 1.282547 & 3 & 6 & 5.541667 & 1.102533 & 4 & 7
\end{tabular}




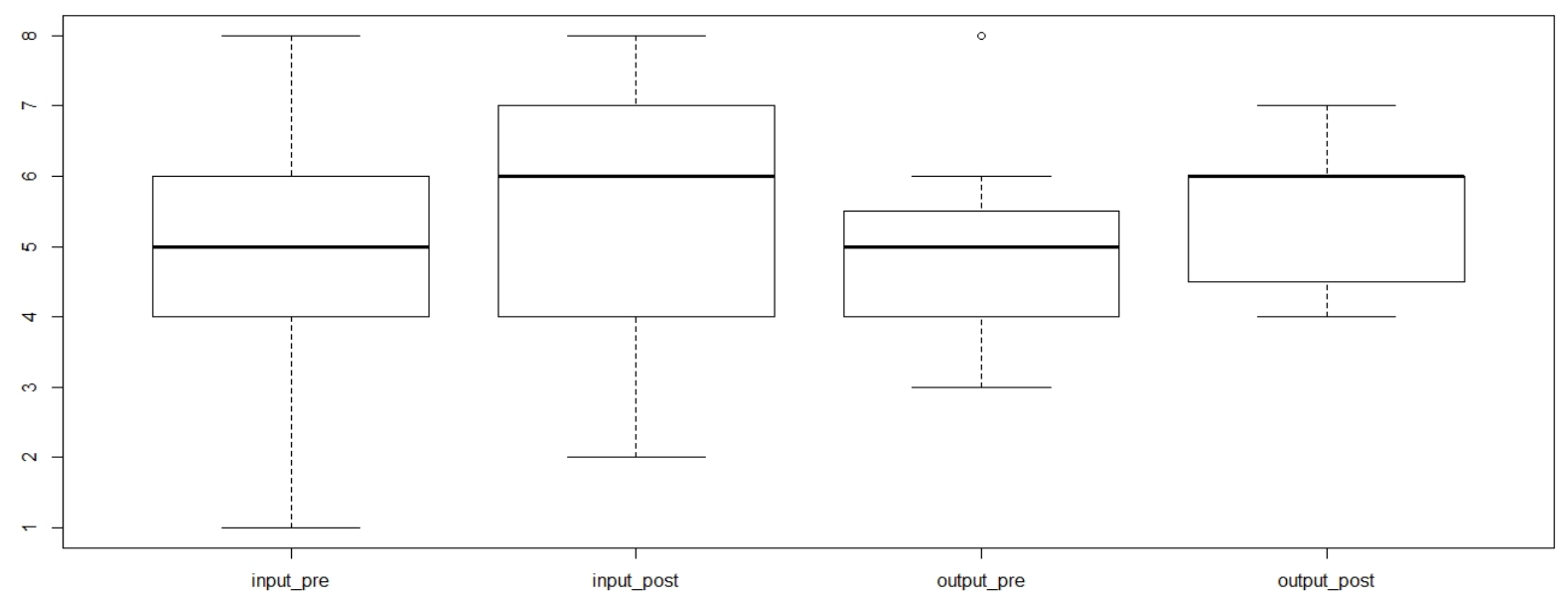

Figure 1 Graphic Comparison of Input Group and Output Group

The difference in sample size between the two groups should be noted here. Input group has almost twice size as output group, but input group shows larger SDs than the output group in both in the pre-test and the post-test. This means that the input group had a wider variety of levels from the outset.

Also, a practice effect of pre-test on post-test should be considered because it could cancel the moderate increases between the two tests. However, the size of this effect is limited since the two tests were conducted with a sufficient interval of approximately three months.

\section{Conclusion}

The results of this study lead to two outcomes. First, soft-CLIL practices improve students' cognitive ability, whether those teaching practices are input-focused or output-focused. However, the effects of the teaching practices under the current study are quite moderate compared to the reviewed literature (Kashiwagi et al., 2019). Therefore, the further question should be like, how Soft-CLIL is implemented for a larger effect.

Second, the effect sizes of input- or output-focused instructions are not different. This could be because the classroom practices became closer to each other against the initial design of the study; for instance, input group was given discussion tasks, while output group listened and read supplemental materials in output tasks.

A possible limitation of this study is that GJTs were conducted with a small number of items. However, more serious one is that the scope of the GJTs was narrowed down to the cause-result relationship and did not include a wider variety of logical connections. Beyond this, a full range of thinking skills in Bloom's taxonomy should be examined in order to explore the relationship of language learning and cognitive development in general.

\section{References}

Bentley, K. (2010). The TKT course CLIL module. Cambridge, United Kingdom: Cambridge University Press. 
Bybee, J. L., \& Beckner, C. (2010). Usage-based Theory. In Bernd Heine and Heiko Narrog (eds), The Oxford Handbook of Linguistic Analysis (pp. 827-856). Oxford: Oxford University Press.

Cleary, K., Nozaki, Y., \& Matsumoto, K. (2012). Science in Focus. Tokyo: Seibido.

Coyle, D., Hood, P., \& Marsh, D. (2010). Content and Language Integrated Learning. Cambridge, United Kingdom: Cambridge University Press.

Dale, L., van del Es, W., \& Tanner, R. (2010) CLIL Skills Leiden: Expertisecentrum mvt, ICLON, Universiteit Leiden. 2010

Dalton-Puffer, C. (2007). Discourse in Content and Language Integrated Learning (CLIL) classrooms. John Benjamins Publishing Company.

Deller, S., \& Price, C. (2007). Teaching other subjects through English. Oxford: Oxford University Press.

Ellis, R. (1991). Grammatically judgments and second language acquisition, Studies in Second Language Acquisition, 13 (2), 161-186.

Godfroid, A., Loewen, S., Jung, S., Park., J., Gass, S., \& Ellis, R. (2015). Timed and untimed grammaticality judgments measure distinct types of knowledge: Evidence from eye-movement patterns, Studies in Second Language Acquisition (37), 269-97.

Kashiwagi, K., \& Kobayashi, U. (2019). Science in CLIL in a Japanese upper secondary school: Focusing on increasing procedural knowledge with a Usage-based Model perspective. J-CLIL, 1, 19-41.

Krathwoal, D. R. (2002). A revision of Bloom's taxonomy: A revision. Theory into Practice, 41 (4), 212-218.

Lin, A. (2019). Beyond soft CLIL/hard CLIL: Charting out programme options along the continuum. Plenary Talk at the $2019 \mathrm{~J}$-CLIL Annual Bilingual Confernce, Tokyo.

Phillips, T., Hitomi, K., \& Yubune, E. (2005). Technical English. Tokyo: Seibido.

Tomasello, M. (1999). The cultural origin of human cognition. Cambridge, MA: Harvard, UP.

Tomasello, M. (2000). First steps toward a usage-based theory of language acquisition. Cognitive Linguistics, 11(1-2), 61-82. 